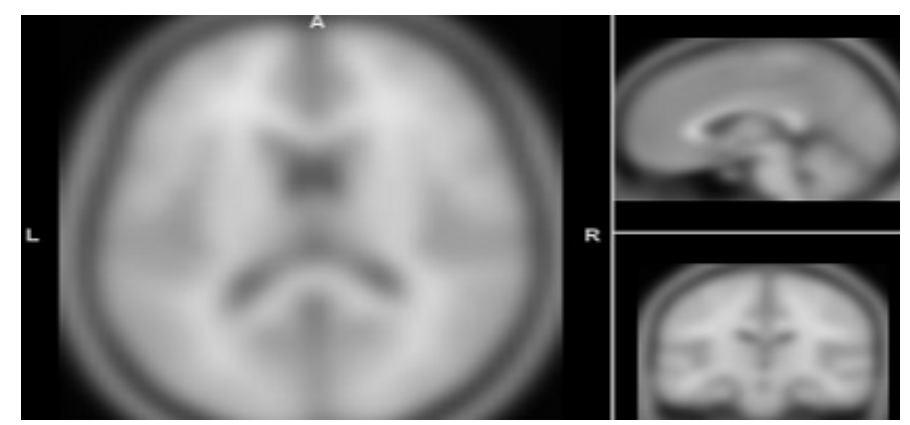

\title{
A Concise Study of Schizophrenia and Resting-state fMRI data analysis
}

\author{
Indranath Chatterjee ${ }^{1}$, Khushboo Mittal $^{2}$ \\ 1 Tong myong University \\ 2 University of Delhi
}

\section{Abstract}

Schizophrenia is a severe mental illness in which people experience hallucinations and delusions. Reasons for happening schizophrenia are genetics, occur in a family, and it is heritable in nature. It can happen due to inappropriate development of the brain, due to complications at the time of birth, due to intake of drugs and an increase in the stress level in the environment. To diagnose critical psychological disorders like schizophrenia, functional magnetic resonance imaging ( $f M R I$ ) is widely used. This technique measures the indirect neural activity of the brain tracing the oxygenation level of the blood flow. Resting-state fMRI measures the same in the rest condition of the patient while doing no cognitive task. In this review article, firstly, we describe schizophrenia and a brief description of its occurrence, cause, symptoms, and treatment. Secondly, we describe the fMRI technology followed by the resting-state analysis and finally, we describe briefly the methods of data analysis to diagnose schizophrenia with resting-state fMRI. This review article may act like a start-up for the research in this domain.

\section{Introduction}

\subsection{Schizophrenia}

Schizophrenia is a chronic, severe mental disorder in which people live in delusions and 
hallucinations ${ }^{[1]}$. The person starts losing its real-world connections. Although it is not a common disorder, it is the most chronic and disabling. It generally origins in childhood and experienced again and reinforced in a later period of life ${ }^{[2]}$. It can happen, including:

- Genetics (heredity) ${ }^{[3]}$

- Brain chemistry and circuits

- Brain abnormality

- Environment

There are no laboratory tests to specifically diagnose schizophrenia. Whenever there are the below-mentioned symptoms present in the persons, then they can be diagnosed by it $^{[4]}$ :

- Delusions

- Hallucinations

- Disorganized speech

- Disorganized or catatonic behavior

Addressing the issue of schizophrenia involves the development of a stress-free environment, a sense of hope, self-reliance, and a personalized awareness of current strengths and challenges ${ }^{[5]}$.

\subsubsection{Occurrence of schizophrenia}

Schizophrenia is a severe mental illness that affects a huge amount of the population worldwide. The prevalence of the disorder is growing high. Around 1\% of the global population is being affected by schizophrenia. It is affected by people regardless of race, ethnicity, geog raphical location, etc. In 2000, the World Health Organization (WHO) found that the prevalence and incidence of the illness are similar across the globe. According to a recent survey, it is found that around 1.2\% of Americans are affected by schizophrenia\#. According to the data released for schizophrenia by WHO in 2004, it was found that the age-standardized DALY (disability-adjusted life-years) rate of India was 286.903 per 100,000 inhabitants.

Schizophrenia can occur in people all through their lifetime, but new instances of the illness are most likely to happen in early adulthood. It occurs mostly in the late adolescent years (the early twenties) to early adulthood (early thirties). People with schizophrenia starts experiencing the basic symptoms during the 16 to 25 years of age. It was found that the men tend to develop the condition for the disorder earlier than the women i.e. early diagnosis is more frequent in males than females. The onset of the illness is rare in 
the older population, as in childhood.

\subsubsection{Reasons for Schizophrenia}

It's very difficult to determine the causes of schizophrenia. Some studies suggest schizophrenia can be caused due to some failure, sorrow, pain, genetic variants, social stress, etc. A combination of different factors such as physical, genetic, psychological and environmental can cause a person to develop the illness.

Some factors that increase the risk for development of the illness may include:

\subsubsection{Genetics}

Schizophrenia is sometimes found to occur in families. It is heritable in nature, but a single gene is not responsible for the circulation of the illness. A different combination of various genes is more likely to be vulnerable to the condition. Researchers have identified 108 genes that are someway associated with the risk of schizophrenia. Most of the identified genes are related to dopamine receptors, glutamate transmission, immune system, and synaptic plasticity. It was also found that it is twin concordance i.e. if one of the identical twins suffers from schizophrenia, the other one will have a 50\% chance of development of the disorder, even if the children are raised separately. However, the genetic factor is not only the reason behind the illness.

\subsubsection{Development of the brain}

The differences in the structure of the brain when studied on people with schizophrenia, suggest that the illness may also occur due to different development of the brain. But the developmental changes may be noticed in people without any mental illness.

\subsubsection{Neurotransmitters}

Neurotransmitters are endogenous chemicals that help in the neurotransmission of information. It is found that the drugs that change the level of neurotransmitters often diminish some symptoms of schizophrenia. It shows that the neurotransmitters may have some connections with the illness. Schizophrenia may be caused by an alteration in the level of dopamine and serotonin or sometimes may be caused due to an imbalance of these two neurotransmitters.

\subsubsection{Birth time complications}

Research suggests that experiencing birth time complications may affect the person to develop the condition for schizophrenia. During and before birth complications such as low birth weight, premature labour, asphyxia during birth, are more likely to affect the 
person on their brain development.

\subsubsection{Social and personal triggers}

Triggers are the different factors that are mostly responsible for the cause of schizophrenia. The triggers include stress such as in job and relationship; emotional, physical and sexual abuse. These stressful situations are not necessarily causing schizophrenia but can trigger their development.

\subsubsection{Drug Intake}

Studies show different drugs such as cannabis, cocaine or LSD may affect the human brain to trigger the development of mental illness. Although, drugs don't cause schizophrenia directly.

\subsubsection{Types of Schizophrenia}

In recent days, schizophrenia is considered a neurodevelopmental disorder. In this mental illness, the patients suffer from various symptoms. According to DSM-5 criteria ${ }^{[6]}$, if two of the following five main symptoms persist, then the person is said to have schizophrenia.

The main symptoms are:

\subsubsection{Delusions:}

Delusions are false beliefs out of reality. The patient thinks several fake thoughts such as he has been harmed or harassed; he gains sudden exceptional fame; some kind of catastrophe may happen. It is very common in schizophrenia.

\subsubsection{Hallucinations:}

Hallucinations can be visual or auditory. In auditory hallucination, the patient use to listen to certain indistinct voices, noise, someone's cry or call for him, which do not exist in actual. Visual hallucinations cause the patient to see unwanted things, some known/ unknown person who does not exist. The person with schizophrenia has a full force in these beliefs.

\subsubsection{Disorganized thinking (speech):}

Disorganized thinking is also a common symptom in schizophrenia. It is basically inferred from disorg anized speech. The patient suffers from the illness experience the impaired ability of effective communication. In rare cases, the patient uses a combination of meaning less words that may not be understood. 


\subsubsection{Disorg anized or abnormal motor behavior:}

In some cases of schizophrenia, the patient shows abnormality in their motor behavior in several ways such as childlike silliness, resistant to any instructions, inappropriate postures, and unresponsiveness to queries.

\subsubsection{Negative symptoms:}

Negative symptoms lead to an inability to function daily life properly. The patients suffering from negative symptoms show a lack of interest in daily activities, withdraw themselves from society and social gatherings, show a lack of emotions, and even show negligence to personal hygiene. Negative symptoms often are seen much before the occurrence of positive symptoms.

\subsubsection{Early symptoms or prodrome of Schizophrenia} Most patients having schizophrenia (around $80 \%$ - $90 \%$ ) have a prodrome which is characterized by some attenuated or subthreshold symptoms appear along with the delusion and hallucination ${ }^{[7]}$. The basic prodromal symptoms include perplexity, the diminished power of understanding, hearing a few indistinct noises, some highly unusual beliefs, guardedness, etc.

According to some studies, the prodromal phase can vary from person to person but it stays for almost one year for each patient. Most of the schizophrenic patients seek help and seemed to be distressed while in Clinical High Risk (CHR) state.

The major classification of schizophrenia was made by Bleuler. He recognized two major classes of the symptoms, viz. positive and negative symptoms. In 1980, Crow suggested that schizophrenia can be classified into two types, referred to as type1 and type2.

The Characteristics of type1 Schizophrenia:

1. Positive Symptoms

2. Normal brain structure

3. Comparitively good response to treatment

4. Dopamine was assumed to be responsible for the neurochemical process that governed the disease.

The Characteristics of type2 Schizophrenia:

1. Negative symptoms 
2. Some abnormalities in brain structure observed on the Computed Tomography scan.

3. Relatively poor responses to the treatment

4. Impaired cognitive function

The type1 schizophrenia with positive symptoms was named as Positive Schizophrenia and the type2 with neg ative symptoms was named Negative Schizophrenia. In 1982 Andreasen suggested ${ }^{[8]}$, that some of the people have both positive and negative schizophrenic symptoms, based on that She added one more type i.e. mixed type along with the previous two.

The symptoms that mainly lie in the two types of schizophrenia are:

\section{Positive Schizophrenia:}

-At least one of the below was present:

1) Hallucinations (auditory, visual, haptic, or olfactory) ${ }^{[8]}$

2) Delusions

3) Positive formal thought disorder

4) The repeated occurrence of disorg anized behaviour.

\section{Negative Schizophrenia:}

-At least two of the below were present to a marked degree:

1) Affective flattening;

2) Anhedonia Asociality (e.g., Not able to feel intimacy or experience pleasure in social contacts);

3) Avolition-apathy (e.g.,impersistence, anergia at work);

4) Attentional impairment.

5) Alogia (e.g., poverty of speech, poor content of speech) ${ }^{[8]}$;

\section{Mixed Schizophrenia:}

This category incorporated patients who show both positive and negative symptoms or did not fulfill the criteria for either one.

\subsubsection{Treatments of Schizophrenia:}

As Schizophrenia was considered to be a brain disease or a set of disease due to transmission of genetic or prenatal or perinatal insult, the assumption was made that this disorder can't be treated by psychological interventions. Most of the patients are still untreated due to unawareness. 
Treatment is sometimes misguided due to the manifestation of similar symptoms in psychosis. But psychosis is not associated with only schizophrenia, but also with other disorders such as dementia, Parkinson's disease, stroke, brain tumours, drug abuse, and alcohol usage. Schizophrenia is a psychosis primarily associated with delusion and hallucinations.

Schizophrenia patients have premature death around 14.5 years lesser than the average mortal age of the normal population. It is found that $40 \%$ of premature deaths are due to suicides. The mean age of hospitalization.

\subsubsection{The process of treatment:}

The goals of treatment of schizophrenia patients are mainly targeting to reduce the symptoms, preventing the relapse of the symptoms, and enhancing the basic functionality, helping the patients to live a normal social life.

Till the mid-1900s, no drugs for schizophrenia were available. The treatment was mostly like confining the patients in asylums and powerful doses of sedative drugs were administered to suppress the behavioral changes. The electric shock treatment also known as electro-convulsive therapy (ECT) was very common to control the depression and psychotic abnormal behaviors. The application of ECT is still controversial and used today for serious psychotic administration.

The process of treatment of schizophrenia can be broadly categorized into two types, namely non-pharmacological therapy and pharmacological therapy. Pharmacotherapy being the necessary thing in treating schizophrenia, the residual symptoms still may remain active. Thus, non-pharmacotherapy like psychotherapy is important. Psychotherapy may be again categorized into three namely,

1. Individual-level therapy
a. Counseling
b. Personal therapy

2. Group level
a. Social therapy

3. Cognitive Therapy

a. Cog nitive behavioural therapy (CBT)

b. Cog nitive enhancement therapy (CET) 
Other psychotherapy include social skills training, rehabilitation, family education, joining self-help groups.

\section{Pharmacological therapy}

Pharmacotherapy involves the administration of medicine. Medication is the most vital form of treating schizophrenia. Antipsychotic medications are the common drugs prescribed to schizophrenia patients. They can control and reduce the symptoms like hallucinations and delusions by affecting the brain neurotransmitters. The dosage and selection of the drug is a very crucial task in antipsychotic medication. It is always targeted to manage the illness with the lowest possible dose. It normally takes 1-2 weeks to start working.

As the medicines for schizophrenia have serious side-effects, patients sometimes become hesitant to take it. Thus, it is also important to support and help the patients to bring their willing ness to cooperate with the process of treatment. Recently, a new treatment option is an available alternative to oral medication in the form of injection. It is called long-acting treatment (LAT) or long-acting injectable (LAI) which can be administered once every few weeks to once every few months depending on the severity providing long term effects on the body.

There are mostly two types of antipsychotic medicines, first-generation, and second generation*. The first-generation drugs show frequent and potential side effects in the brain, sometimes causing temporary or permanent motion disorder. First-generation antipsychotic drugs include ${ }^{[9][10]}$ :
a. Chlorpromazine
b. Fluphenazine
c. Haloperidol
d. Perphenazine

These drugs are comparatively cheaper than the second generation. Thus, these drugs may be administered to patients when long-term treatment is needed.

The second-generation antipsychotics are generally recommended and prescribed due to its less side-effects in the brain. Second-generation antipsychotics include ${ }^{[9][10]}$ :
a. Aripiprazole (Abilify)
b. Asenapine (Saphris) 
c. Brexpiprazole (Exultar)

d. Cariprazine (Vraylar)

e. Clozapine (Clozaril)

f. Iloperidone (Fanapt)

g. Lurasidone (Latuda)

h. Olanzapine (Zyprexa)

i. Paliperidone (Invega)

j. Quetiapine (Seroquel)

k. Risperidone (Risperdal)

I. Ziprasidone (Geodon)

Alternate ways of treatment include:

Hospitalization

For severe cases, when periods of symptoms become very frequent and the patients start showing abnormal behaviour, hospitalization may be prescribed to provide proper nutrition, adequate sleep, and safety.

\section{Electroconvulsive therapy}

When the adult schizophrenic patients stop responding to medication, electroconvulsive therapy (ECT) may be prescribed in rare cases. ECT may pose helpful for patients suffering from depression too.

While a huge number of schizophrenia patients are still untreated due to social exclusion and stigma having negative stereotypes, lifelong treatment including medication and psychotherapy is required in schizophrenia, even after subsiding of symptoms. Proper dosage of medications and psychosocial therapy can help in the management of the disorder.

\subsection{FMRI (Functional Magnetic Resonance Imaging)}

FMRI stands for functional magnetic resonance imaging. It is an imaging technique used to identify the functional activations across different regions in the brain ${ }^{[11]}$. The fMRI data are 4-D data which consist of 3-Dimensional images over time ${ }^{[1]}$. A 3-D data is a sequence of 2-Dimensional images (or slices) over the entire brain. Each slice consists of brain volume small units which are called a voxel. Therefore, a specific spot of the brain is represented by voxe ${ }^{[12]}$. So, by comparing the fMRI data of schizophrenia patients and healthy control we can diagnose the disease, by detecting the regions which are active 
during a particular task in schizophrenia patients as well as in healthy controls ${ }^{[12]}$.

\subsubsection{Principal of FMRI}

When neurons get activated, by the process of the hemodynamic response, they are supplied with raised cerebral blood flow and the oxygen supply usually more than they required, by the adjacent capillaries. They do not have any internal set aside of energy, neither in the form of oxygen nor glucose. On the bases of their distinctive magnetic susceptibilities, MRI detects the activations by measuring the ratio of the levels of oxyhemoglobinand deoxyhemoglobin. This technique is called blood oxygen leveldependent (BOLD) imaging. According to the recent researches, BOLD signals intent by arteries partial pressure of both $\mathrm{O} 2$ and $\mathrm{CO} 2$. These BOLD signals are the main foundation of $\mathrm{fMRI}$, through which we can construct maps of active brain regions which are active during a particular task or reacting to a detectable change in the neural activity at a low frequency $(0.01-0.01 \mathrm{~Hz})^{[13]}$.

\subsubsection{Why do we use fMRI}

Because of its excellent spatial resolution, we use fMRI, from which we can detect the regions of the brain which are active during the tasks ${ }^{[14]}$.

\subsection{Resting-state fMRI (rs-fMRI)}

Resting-state fMRI is widely used for mental, neurological, neuropsychiatric disorders. Unlike task-based fMRI, rs-fMRI does not prescribed subjects to do any cognitive (mental, emotional) task. The spontaneous neural activities can be related by lowfrequency oscillations $(0.01 \mathrm{~Hz}-0.1 \mathrm{~Hz})$ of $r s-f M R I$.

We can perform rs-fMRI data analysis in many ways, with popularly known techniques like ICA and seed-based analysis but before that, we have to do preprocessing of data by reorganizing and eliminating the confounding artifacts. Moreover, in these preprocessing steps, rs-fMRI data requires correction of slice-timing, spatial filtering, motion compensation, and normalization.

\subsubsection{Challenges of rs-fMRI}

The removal of noise, i.e, temporal filtering must be done carefully so that there is no removal of appropriate or closely connected low-frequency resting-state signals. Noise levels from movement and physiological (like breathing) needs in rs-fMRI are very high. There are less than $5 \%$ of total signals which derive from neurological activity. The main problems for rs-fMRI studies are the frequencies of cardiac and respiratory pulsations 
which are close to the resting state networks ${ }^{[3]}$.

\subsubsection{Methods of analysis of rs-fMRI data}

A large amount of rs-fMRI data can be easily compared with the help of graphs and maps. The analysis of rs-fMRI data can be done by different methods and models, like Amplitude of Low Frequency Fluctuations (ALFF), fractional ALFF, Regional Homogeneity (ReHo), Functional connectivity Density, Seed- Based Functional connectivity Analysis or ROI-based FC, Independent Component Analysis (ICA $)^{[13]}$.

The ALFF methods measure the strength of the BOLD signals within the range of lowfrequency between $0.01 \mathrm{~Hz}$ and $0.1 \mathrm{~Hz}$. Both fractional-ALFF and ALFF reveal the densities of the active brain regions. Both of them do not provide details about the functional connectivity within regions of the brain. These methods are known for their simplicity of analysis without any hypothesis. Both of them give long-term test-retest reliability and high temporal stability.

ReHo analysis is a voxel-based method with providing information about the different features of regional neurological activity, it finds the correlation between the BOLD signal of a given voxel and its close by neighbours. It is calculated by the Kendall Coefficient of corresponding BOLD signals. The higher value of ReHo represents centrality and higher coherence of regional brain activity.

Seed based Functional connectivity is also called ROI-based functional connectivity, it finds the regions which are active at the same time when the seed region is active. It visualizes the correlation between seed and the rest of the brain regions by the connectivity matrix, which shows the strength of all connections. These regions may not be directly connected yet the coupling of activation of different brain regions shows their involvement in the same functional process and thus indicates that they are functionally connected.

Independent component analysis (ICA) is used to separate spatially independent figures ${ }^{[15]}$ from their unswerving mixed blood-oxygen-level-dependent (BOLD) signals in the form of spatial maps, which are temporally correlated ${ }^{[16]}$.

\subsubsection{Need rs-fMRI}

- Infants, neuropsychiatric disordered patients cannot able to perform the task in task- 
based fMRI. rs-fMRI possess no burden on them to perform any task.

- They provide information about the neural activities or active regions, free from demanding external stimuli.

- The rs-fMRI is capable of finding a marker to disting uish an individual with major depressive disorder (MDD) from a healthy control ${ }^{[17]}$.

\subsubsection{Advantages of rs-fMRI}

Unlike task-based fMRI, rs-fMRI can be easily performed by infants, young children and a person who is suffering from neurological and psychiatric diseases. Resting-state functional connectivity determines tightly coupled functional networks of the brain by using spontaneous synchronized fluctuations in the BOLD signal. These spontaneous fluctuations are postulated to act to coordinate, org anize, and maintain functional brain systems ${ }^{[18]}$. Now, rs-fMRI is placing a minimum mental burden on the participants and taking a nominal time of a few minutes only.

\section{Conclusions and future scope}

In this article, we have discussed first about schizophrenia, its symptoms, causes, and treatments. Secondly, about fMRI followed by the rs-fMRI, and finally, we have described the methods of data analysis of rs-fMRI and its advantages over task-based fMRI. This paper throws light on the applicability of rs-fMRI for diagnosis of schizophrenia. As a scope of future work, researcher after gaining basic knowledge on the disorder and fMRI, may study the different pathophysiological aspects of schizophrenia using rs-fMRI.

\section{Footnotes:}

[\#] Source Website link: https://www.mentalhelp.net/schizophrenia/statistics/

[*] Source Web link: https://ce.mayo.edu/sites/ce.mayo.edu/files/Friday_0800_Hu.pdf

\section{References}

1. ${ }^{a}, \mathrm{~b}$ Indranath Chatterjee. (2018). Mean deviation based identification of activated voxels from time-series fMRI data of schizophrenia patients. F1000Res, vol. 7, 1615. doi:10.12688/f1000research.16405.2.

2. 'Arieti, Silvano. (1955). "Interpretation of schizophrenia".

3. ${ }^{a, b}$ Kallmann, Franz Josef. (1938). "The genetics of schizophrenia".

4. ^ Nancy C. Andreasen. (1995). Symptoms of Schizophrenia. Arch Gen Psychiatry, vol. 52 (5), 341. doi:10.1001/archpsyc.1995.03950170015003. 
5. ^ Lysaker, Paul H., and Kelly D.. (2008). "Is Recovery from Schizophrenia Possible? An Overview of Concepts, Evidence, and Clinical Implications". Buck Primary Psychiatry 15.6.

6. ^ (2013). Diagnostic and Statistical Manual of Mental Disorders. American Psychiatric Publishing, Incorporated.

7. ^ A. R. Yung, P. D. McGorry. (1996). The Prodromal Phase of First-episode Psychosis: Past and Current Conceptualizations. Schizophrenia Bulletin, vol. 22 (2), 353-370. doi:10.1093/schbul/22.2.353.

8. a, b, c Nancy C. Andreasen. (1990). Positive and Negative Symptoms in Schizophrenia. Arch Gen Psychiatry, vol. 47 (7), 615. doi:10.1001/archpsyc.1990.01810190015002.

9. a, b Stefan Leucht, Caroline Corves, Dieter Arbter, Rolf R Engel, Chunbo Li, John M Davis. (2009). Second-generation versus first-generation antipsychotic drugs for schizophrenia: a meta-analysis. The Lancet, vol. 373 (9657), 31-41. doi:10.1016/s01406736(08)61764-X.

10. a, b Patel, Krishna R., et al.. (2014). "Schizophrenia: overview and treatment options". Pharmacy and Therapeutics.

11. ^Indranath Chatterjee, Virendra Kumar, Sahil Sharma, Divyanshi Dhingra, Bharti Rana, Manoj Agarwal. (2019). Identification of brain regions associated with working memory deficit in schizophrenia. F1000Res, vol. 8, 124. doi:10.12688/f1000research.17731.1.

12. a, b Indranath Chatterjee, Manoj Agarwal, Bharti Rana, Navin Lakhyani, Naveen Kumar. (2018). Bi-objective approach for computer-aided diagnosis of schizophrenia patients using fMRI data. Multimed Tools Appl, vol. 77 (20), 26991-27015. doi:10.1007/s11042-018-5901-0.

13. a, b H. Lv, Z. Wang, E. Tong, L.M. Williams, G. Zaharchuk, M. Zeineh. (2018). RestingState Functional MRI: Everything That Nonexperts Have Always Wanted to Know. AJNR Am J Neuroradiol. doi:10.3174/ajnr.a5527.

14. `Judith M. Ford, BrianJ. Roach, Vanessa A. Palzes, Daniel H. Mathalon. (2016). Using concurrent EEG and fMRI to probe the state of the brain in schizophrenia. Neurolmage: Clinical, vol. 12, 429-441. doi:10.1016/j.nicl.2016.08.009.

15. ^ Chatterjee, Indranath. (2018). GICA supported region-based feature selection technique for fMRI data. Zenodo.

16. ^ Erik Barry Erhardt, Srinivas Rachakonda, EdwardJ. Bedrick, Elena A. Allen, Tülay Adali, Vince D. Calhoun. (2010). Comparison of multi-subject ICA methods for analysis of fMRI data. Hum. Brain Mapp., vol. 32 (12), 2075-2095. doi:10.1002/hbm.21170. 
17. ^ Runa Bhaumik, Lisanne M. Jenkins, Jennifer R. Gowins, Rachel H. Jacobs, Alyssa Barba, Dulal K. Bhaumik. (2017). Multivariate pattern analysis strategies in detection of remitted major depressive disorder using resting state functional connectivity. Neurolmage: Clinical, vol. 16, 390-398. doi:10.1016/j.nicl.2016.02.018.

18. ^ Lucina Q. Uddin. (2010). Typical and atypical development of functional human brain networks: insights from resting-state fMRI. Front. Syst. Neurosci., vol. 4. doi:10.3389/fnsys.2010.00021. 